\title{
LA TUTELA JUDICIAL DIFERENCIADA, UN CAMINO PARA EL EFECTIVO GOCE DE LOS DERECHOS
}

\section{DIFFERENTIATED JUDICIAL PROTECTION, A WAY FOR EFFECTIVE ENJOYMENT OF RIGHTS}

\section{María Natalia Juez}

DOI: https://doi.org/10.37767/2591-3476(2020)36

\section{Comentario a}

P. B., G. T. C. Swiss Medical Group - Amparo-Expte. N8268042

Cámara 4ta de Apelaciones en lo Civil y Comercial

(Poder Judicial de la Provincia de Córdoba)

\section{Disponible en}

https://bit.ly/2E68GCY

\section{RESUMEN:}

El reconocimiento de derechos debe ir acompañado de acciones positivas del Estado que aseguren su eficacia y tomen en especial consideración las diferentes realidades y circunstancias de los justiciables, lo que debe ser un pauta de interpretación que impregne sus decisiones.

\section{ABSTRACT}

The recognition of rights must be accompanied by positive actions by the State that ensure its effectiveness and take special consideration of the different realities and circumstances of the citizens, which should be a guideline of interpretation that permeates their decisions.

PALABRAS CLAVE: Tutela judicial diferenciada; personas en condición de vulnerabilidad; tutela anticipada; discapacidad; derecho a la salud.

KEY WORDS: Differentiated judicial protection; people in vulnerable condition; early guardianship; disability; right to health. 


\section{Introducción}

En marco de una acción de amparo, los padres de la niña G.T.P.B. reclaman a SWISS MEDICAL GROUP la provisión de dos audífonos marca Widez Modelo U2 + FM + SCOLA $\mathrm{REACH}+\mathrm{DEX}+\mathrm{FEX}$, lo cual es solicitado también como medida cautelar. En contra del acogimiento favorable de esa medida se levanta la demandada ante lo cual la Excma. Cámara de Apelaciones Civil y Comercial de $4^{\circ}$ nom. de la ciudad de Córdoba confirma lo resuelto por el tribunal de primera instancia. Para así decidir se valoran los elementos de la causa a la luz la pertenencia de la niña a grupos en situación de vulnerabilidad y la entidad de los derechos en juego como el derecho a la salud y a la educación.

\section{El progresivo reconocimiento de derechos como primer paso para su goce.}

A los largo de los años hemos sido testigos de un proceso de progresivo reconocimiento de derechos y garantías a la ciudadanía, el que en nuestro país ha venido de la mano de la Reforma Constitucional del año 1994 y la denominada constitucionalización de los Tratados Internacionales. Más recientemente, con la promulgación del Código Civil y Comercial de la Nación en el año 2015 se avanzó hacia la convencionalización del derecho privado.

Relacionado a la situación fáctica de la que parte el fallo comentado, podemos referir que el Pacto internacional de Derechos económicos, sociales y culturales dispone en su art. 12 que "los Estados partes en el presente Pacto reconocen el derecho de toda persona al disfrute del más alto nivel posible de salud física y mental". Por otro lado, bajo la denominación derecho a la preservación de la salud y al bienestar, el artículo 11 de la Declaración Americana sobre Derechos y Deberes del Hombre prescribe que "toda persona tiene derecho a que su salud sea preservada por medidas sanitarias y sociales, relativas a la alimentación, el vestido, la vivienda y la asistencia médica, correspondientes al nivel que permitan los recursos públicos y la comunidad". La Convención sobre los Derechos de las Personas con Discapacidad dice que "los Estados Partes reconocen que las personas con discapacidad tienen derecho a gozar del más alto nivel posible de salud sin discriminación por motivos de discapacidad. Los Estados Partes adoptarán las medidas pertinentes para asegurar el acceso de las personas con discapacidad a servicios de salud que tengan en cuenta las cuestiones de género, incluida la rehabilitación relacionada con la salud".

En consonancia con estos tratados, se han dictado a nivel nacional leyes de protección integral de las personas con discapacidad como la N²2.431 y la 24.901.

Sin embargo, este importante y progresivo fortalecimiento en el elenco de derechos y garantías, ponen en evidencia la necesidad de que el Estado asegure los mecanismos necesarios para que esas normas no sean meras enunciaciones y puedan concretarse en la efectivo goce de esos derechos.

\section{El goce de los derechos como meta.}

El reconocimiento de los derechos en la letra de la ley, si bien es un importante avance no deja de ser simplemente el primer paso. Debe necesariamente ser complementado con acciones tendientes a su concreción, cual es el efectivo goce de esos derechos.

En otras palabras, es necesario articular los medios para que el Estado no se evidencie como incapaz de asegurar esos derechos. Es así como el tradicional principio de la de la tutela judicial efectiva adquiere especiales matices derivados de la necesidad de demos- 
trar que el sistema judicial puede y debe adaptarse a las necesidades de la ciudadanía. No basta ya con tener acceso a reclamar ante la justicia u obtener una resolución judicial como respuesta al requerimiento formulado, es necesario que el actuar del órgano judicial asegure la operatividad de los derechos y garantías reconocidos por las normas.

El énfasis debe estar puesto en la eficacia de la función jurisdiccional y no en apego a las formas, teniendo presente que las formas y mecanismos procesales no son sino un medio para la concreción de los derechos sustanciales.

Es aquí en donde entra en escena la tutela judicial diferenciada que impone la aplicación de mecanismos que permitan hacer efectivos los derechos y garantías consagrados en los instrumentos internacionales y en las normas, con especial atención en los grupos más vulnerables.

Al decir de Peyrano estamos ante una tutela procesal diferenciada "cuando -excepcionalmente y a raíz de experimentar urgencias apremiantes el requirente del servicio de justicia o de las singularidades del derecho material cuya aplicación se reclama- se hubiera instrumentado un montaje procesal autónomo de cierta complejidad, portador de una pretensión principal y que cuenta con la dirección de un órgano jurisdiccional investido de facultades incrementadas e inusuales; estructura que deberá satisfacer, en la medida de lo razonable, la garantía del debido proceso (que ampara tanto al requirente del servicio de justicia como al requerido) y que se deberá apartar, en varios aspectos, y, notoriamente, de las matrices vigentes clásicas. Dicho montaje procesal deberá brindar al demandante un trato preferencial y admitir, por lo común, una legitimación activa amplia". (Peyrano, 2009: p.22).

La heterogeneidad de la situación en que se encuentran las personas, obligan a los operadores de justicia a abandonar clasificaciones y/o mecanismos estructurados y estancos a la hora de resolver. Deben, por el contrario, valorar las particularidades de cada caso y situación, para en base a ellas elaborar una respuesta que se evidencie efectiva. No puede permitirse que el sistema judicial actúe de manera ineficaz cercenando o limitando los derechos que la Constitución reconoce.

Conjuntamente con el fenómeno de reconocimiento de derechos, en el último tiempo se ha ido tomando conciencia sobre los obstáculos que muchas personas deben enfrentar para acceder a la justicia y lograr el ejercicio de sus derechos, lo que muchas veces está vinculado con pertenecer a sectores que se encuentran en situación de vulnerabilidad.

Especial relevancia sobre la cuestión tienen las Reglas de Brasilia sobre el "Acceso a la Justicia de las Personas en condición de vulnerabilidad" , que contienen un conjunto de directrices y recomendaciones para los Poderes Judiciales con miras a modificar sus prácticas y enervar las causas que impiden a las personas en situación de vulnerabilidad acceder a una tutela judicial efectiva. Allí se considera en condición de vulnerabilidad a "aquellas personas que, por razón de su edad, género, estado físico o mental, o por circunstancias sociales, económicas, étnicas y/o culturales, encuentran especiales dificultades para ejercitar con plenitud ante el sistema de justicia los derechos reconocidos por el ordenamiento jurídico". Dentro de la enunciación de causas de vulnerabilidad se mencionan la edad (niñez o ancianidad) y la discapacidad física, mental o sensorial, permanente o transitoria. 
Por su parte la CSJN en el emblemático caso "Halabi" tiene dicho que el acceso a la justicia debe asegurarse en forma diferenciada cuando "exista un fuerte interés estatal en su protección, sea por su trascendencia social o en virtud de las particulares características de los sectores afectados".

\section{El caso.}

Los padres de la niña G.T.P.B. promueven una acción de amparo en contra de SWISS MEDICAL GROUP solicitando la provisión de dos audífonos para su hija que presenta un diagnóstico de hipoacusia. Idéntico objeto tiene la medida cautelar peticionada, la que es despachada favorablemente por el juez de primera instancia. En contra de esa decisión se alza la demandada, quien apela la medida que en definitiva es confirmada por el tribunal de alzada.

En apariencia, nos encontramos ante una resolución que analiza cuestiones meramente procedimentales, vinculadas a los requisitos que deben verificarse para el despacho de una medida como lo es la tutela anticipada.

Así, se advierte que las cuestiones abordadas en la resolución bajo análisis pueden agruparse en dos grande temas. El primero de ellos relacionado con las condiciones en las que fue despachada por el tribunal de primera instancia la medida cautelar solicitada por los actores. La accionada aquí se agravia porque la medida fue dispuesta inaudita parte y porque también su objeto se identifica totalmente con el de la pretensión de fondo de la acción entablada. Al respecto, el tribunal de alzada refiere que el contradictorio se ha verificado en la posibilidad de la apelante de expresar sus agravios, por lo que desaparece al respecto el requisito de trascendencia necesario para reconocer el vicio procedimental que se acusa. En relación a la identidad entre la pretensión final y la cautelar, en una concisa remisión expone que ese aspecto es de la esencia misma de la tutela anticipada y que la medida dispuesta en autos reviste esa naturaleza.

El segundo eje, se relaciona con los requisitos de procedencia de la medida cautelar, y, en especial, su valoración en el caso concreto. Es aquí en donde -en mi opinión- se encuentra la riqueza del precedente. Es que la resolución bajo análisis, se encuentra impregnada de pautas de valoración e interpretación que evidencian la aplicación de la denominada tutela judicial diferenciada.

Por ejemplo, al valorar la verosimilitud en el derecho, se hace referencia a que la prescripción médica que aconseja el uso del audífono y en la que se basa la pretensión, no ha sido reconocida lo que en un esquema tradicional debilitaría su trascendencia valorativa. Sin embargo, encontrándose en juego derechos esenciales como el derecho a la salud y a la educación de una niña, se remarca la instrumentalidad de las formas y se flexibiliza la exigencia.

Refieren expresamente que cuando está en juego la protección a la salud de una persona con discapacidad, la procedencia de una medida cautelar debe ser siempre menos rigurosa pues debe valorarse la entidad del perjuicio que su rechazo podría ocasionar en la persona, que por lo general es mucho mayor que el que genera su concesión en la otra parte. Idéntico razonamiento es el que, en definitiva, habilita el despacho de una tutela anticipada. 
Refuerzan incluso su decisión y pautas de interpretación invocando la existencia de una relación de consumo que también es merecedora de pautas interpretativas diferenciadas en atención a la posición de vulnerabilidad de una de las partes.

Finalmente, en atención al peligro en la demora como requisito formal de procedencia, refieren también que la naturaleza misma de los derechos en juego pone en evidencia el riesgo en la demora. Se valora incluso que tratándose de una niña con una discapacidad auditiva, no sólo se afectaría su derecho a la salud sino también el derecho a la educación, porque por la funcionalidad misma del aparato requerido se infiere su vinculación con el desarrollo de sus actividades educativas.

Vemos entonces que la valoración de cada uno de los elementos en que se sustenta la decisión adoptada, ha estado motivada por las especiales circunstancias del caso concreto, inclinando la balanza conforme los factores de vulnerabilidad que presenta la interesada y la entidad de los derechos en juego. Todo lo cual habilita la aplicación de pautas interpretativas diferenciadas que tienen como norte el efectivo goce de los derechos.

\section{Conclusiones}

Se trata de una resolución breve y concisa, que en apariencia es meramente formal, sin embargo se encuentra impregnada de pautas y principio de valoración que permiten flexibilizar las normas procesales para acercar a la jurisdicción un poco más a realización de su finalidad esencial, cual es la concreción del valor justicia.

En casos como este, la necesidad de implementar criterios valorativos diferenciados se impone con mayor claridad y fuerza a la luz de los derechos tan esenciales en juego, en los que el apego a las normas escritas y tradiciones podrían conculcar el efectivo goce de los derechos.

El diseño de acciones tendientes a consagrar la vigencia de los derechos humanos no es sino el cumplimiento de una manda constitucional, ya que la Carta Magna en su art. 75 inc. 23 menciona como facultad del Congreso "la de legislar y promover las medidas de acción positiva que garanticen la igualdad real de oportunidades y de trato, y el pleno goce y ejercicio de los derechos reconocidos por esta Constitución y por los tratados internacionales vigentes de derechos humanos, en particular respecto de los niños, las mujeres, los ancianos y las personas con discapacidad". El Poder Judicial debe ir en esa misma senda, teniendo siempre como finalidad esencial garantizar el pleno goce y ejercicio de los derechos reconocidos por el ordenamiento jurídico.

\section{REFERENCIAS BIBLIOGRÁFICAS}

- PEYRANO, Jorge W., Precisiones sobre el concepto de tutela diferenciada, Revista de Derecho Procesal, T. 2009-1, Rubinzal-Culzoni, Santa Fe, 2009, p.22. 